\title{
Article
}

\section{A Parametric Type of Cauchy Polynomials with Higher Level}

\section{Takao Komatsu}

check for updates

Citation: Komatsu, T. A Parametric Type of Cauchy Polynomials with Higher Level. Axioms 2021, 10, 207. https://doi.org/10.3390/ axioms10030207

Academic Editor: Jesús Martín Vaquero

Received: 8 August 2021

Accepted: 28 August 2021

Published: 30 August 2021

Publisher's Note: MDPI stays neutral with regard to jurisdictional claims in published maps and institutional affiliations.

Copyright: (C) 2021 by the author. Licensee MDPI, Basel, Switzerland. This article is an open access article distributed under the terms and conditions of the Creative Commons Attribution (CC BY) license (https:// creativecommons.org/licenses/by/ $4.0 /)$.
Department of Mathematical Sciences, School of Science, Zhejiang Sci-Tech University, Hangzhou 310018, China; komatsu@zstu.edu.cn

Abstract: There are many kinds of generalizations of Cauchy numbers and polynomials. Recently, a parametric type of the Bernoulli numbers with level 3 was introduced and studied as a kind of generalization of Bernoulli polynomials. A parametric type of Cauchy numbers with level 3 is its analogue. In this paper, as an analogue of a parametric type of Bernoulli polynomials with level 3 and its extension, we introduce a parametric type of Cauchy polynomials with a higher level. We present their characteristic and combinatorial properties. By using recursions, we show some determinant expressions.

Keywords: cauchy polynomials and numbers; recurrence relations; determinants

MSC: 11B75; 05A15; 05A19; 11C08; 15A15

\section{Introduction}

Let $r \geq 1$ be an integer. For real numbers $p, q$, define bivariate Cauchy polynomials $c_{n}^{(r, i)}(p, q)$ with higher level $(i=0,1, \ldots, r-1)$ as

$$
\frac{t f_{q}^{(r, i)}(t)}{(1+t)^{p} \log (1+t)}=\sum_{n=0}^{\infty} c_{n}^{(r, i)}(p, q) \frac{t^{n}}{n !},
$$

where

$$
f_{q}^{(r, i)}(t)=\sum_{n=0}^{\infty}(-1)^{n} \frac{(q t)^{r n+i}}{(r n+i) !} .
$$

Their complementary polynomials $\widehat{c}_{n}^{(r, i)}(p, q)(i=0,1, \ldots, r-1)$ are defined as

$$
\frac{t \widehat{f}_{q}^{(r, i)}(t)}{(1+t)^{p} \log (1+t)}=\sum_{n=0}^{\infty} \widehat{c}_{n}^{(r, i)}(p, q) \frac{t^{n}}{n !},
$$

where

$$
\widehat{f}_{q}^{(r, i)}(t)=\sum_{n=0}^{\infty} \frac{(q t)^{r n+i}}{(r n+i) !} .
$$

When $q=i=0, c_{n}(x)=c_{n}^{(r, 0)}(x, 0)=\widehat{c}_{n}^{(r, 0)}(x, 0)$ is a Cauchy polynomial, defined by

$$
\frac{t}{(1+t)^{x} \log (1+t)}=\sum_{n=0}^{\infty} c_{n}(x) \frac{t^{n}}{n !}
$$

(cf. [1]). When $p=q=i=0, c_{n}=c_{n}^{(r, 0)}(0,0)=\widehat{c}_{n}^{(r, 0)}(0,0)$ is the classical Cauchy number (cf. $[2,3])$, defined by

$$
\frac{t}{\log (1+t)}=\sum_{n=0}^{\infty} c_{n} \frac{t^{n}}{n !}
$$


The numbers $c_{n}(1)$ are often called Cauchy numbers of the second kind. The sum of products of Cauchy numbers are studied in [4]. Some recurrence relations are studied in [5]. The Cauchy numbers are closely related to the harmonic polynomials, and the Cauchy polynomials are related to the Nörlund polynomials $[1,6,7]$. On the other hand, Cauchy numbers and polynomials are closely related to Bernoulli numbers $B_{n}$ and polynomials $B_{n}(x)$, which are defined by

$$
\frac{t e^{t x}}{e^{t}-1}=\sum_{n=0}^{\infty} B_{n}(x) \frac{t^{n}}{n !}
$$

and $B_{n}=B_{n}(0) . e^{t}-1$ and $\log (1+t)$ are inverse functions, and $c_{n} / n$ ! are often called Bernoulli numbers of the second kind [8]. Bernoulli numbers are expressed in terms of Stirling numbers of the second kind and Cauchy numbers are expressed in terms of Stirling numbers of the first kind [9].

There are many generalizations of Cauchy numbers, including higher-order numbers [10], degenerate Cauchy numbers and polynomials [11,12], poly-Cauchy numbers [9], and hypergeometric Cauchy numbers [13]. They may be analogues of degenerate Bernoulli numbers and polynomials [14], poly-Bernoulli numbers [15], and hypergeometric Bernoulli numbers [16-18], respectively. In this paper, we propose still a different kind of generalization of Cauchy numbers and polynomials.

When $r=1, f_{q}^{(1,0)}=f_{q}^{(1,0)}(t)=e^{-q t}$ and $\widehat{f}_{q}^{(1,0)}=e^{-q t}$. When $r=2, f_{q}^{(2,0)}=\cos q t$ and $\widehat{f}_{q}^{(2,0)}=\cosh q t, f_{q}^{(2,1)}=\sin q t$ and $\widehat{f}_{q}^{(2,1)}=\sinh q t$. When $r=3$,

$$
\begin{aligned}
& f_{q}^{(3,0)}=\frac{e^{-q t}+e^{-q \omega t}+e^{-q \omega^{2} t}}{3}, \quad \hat{f}_{q}^{(3,0)}=\frac{e^{q t}+e^{q \omega t}+e^{q \omega^{2} t}}{3}, \\
& f_{q}^{(3,1)}=\frac{e^{-q t}+\omega^{2} e^{-q \omega t}+\omega e^{-q \omega^{2} t}}{3}, \quad \hat{f}_{q}^{(3,1)}=-\frac{e^{q t}+\omega^{2} e^{q \omega t}+\omega e^{q \omega^{2} t}}{3}, \\
& f_{q}^{(3,2)}=\frac{e^{-q t}+\omega e^{-q \omega t}+\omega^{2} e^{-q \omega^{2} t}}{3}, \quad \hat{f}_{q}^{(3,2)}=\frac{e^{q t}+\omega e^{q \omega t}+\omega^{2} e^{q \omega^{2} t}}{3},
\end{aligned}
$$

where $\omega=\frac{-1+\sqrt{-3}}{2}$ and $\omega^{2}=\bar{\omega}=\frac{-1-\sqrt{-3}}{2}$ are the primitive cube roots of unity.

In [19], by referring to Osler's lemma [20], explicit forms of the two bivariate series involving sin and cos functions are obtained. Then, in [19], a parametric type of Bernoulli polynomials is introduced and their basic properties are studied [21]. More precisely, two kinds of bivariate Bernoulli polynomials are introduced as

$$
\frac{t e^{p t}}{e^{t}-1} f_{q}^{(2,0)}=\sum_{n=0}^{\infty} B_{n}^{(2,0)}(p, q) \frac{t^{n}}{n !}
$$

and

$$
\frac{t e^{p t}}{e^{t}-1} f_{q}^{(2,1)}=\sum_{n=0}^{\infty} B_{n}^{(2,1)}(p, q) \frac{t^{n}}{n !} .
$$

Recently, in [22], three kinds of trivariate Bernoulli polynomials are studied. Such bivariate and trivariate Bernoulli polynomials are called the parametric type of Bernoulli polynomials with levels 2 and 3, respectively. In particular, some determinant expressions of these polynomials are also given.

In [23], two trigonometric extensions of bivariate Euler polynomials were introduced and several properties related to these extensions were established. In [24], two parametrictype families of the Fubini-type polynomials were introduced and studied. In [25], a type of generalized parametric Apostol-Bernoulli, Apostol-Euler, and Apostol-Genocchi polynomial was introduced and their basic properties were studied. In [26], the real and imaginary parts of a general set of complex Appell polynomials can be represented in terms of the Chebyshev polynomials of the first and second kind. 
As an analogous generation of the classical Euler numbers, Lehmer [27] introduced and studied generalized Euler numbers $W_{n}$, defined by the generating function

$$
\frac{1}{f_{1}^{(3,0)}}=\sum_{n=0}^{\infty} W_{n} \frac{t^{n}}{n !} \text {. }
$$

Notice that $W_{n}=0$ unless $n \equiv 0(\bmod 3)$. In [28], more general Lehmer's-type Euler numbers were considered.

In [29], along with ideas about parametric Bernoulli polynomials mentioned above, a parametric type of Cauchy numbers with levels 2 and 3 is introduced and studied. However, such levels can be raised by an extension. In this paper, as an analogue of a parametric type of Bernoulli polynomials with level 3 and its extension, we introduce a parametric type of Cauchy polynomials with a higher level. We give their characteristic and combinatorial properties. By using recursions, we show some determinant expressions.

Since for $i=0,1, \ldots, r-1$

$$
(-1)^{r n+i}= \begin{cases}(-1)^{n+i} & \text { if } r \text { is odd; } \\ (-1)^{i} & \text { if } r \text { is even, }\end{cases}
$$

we see that

$$
\begin{aligned}
& f_{q}^{(r, i)}(-t)= \begin{cases}(-1)^{i} \widehat{f}_{q}^{(r, i)}(t) & \text { if } r \text { is odd; } \\
(-1)^{i} f_{q}^{(r, i)}(t) & \text { if } r \text { is even },\end{cases} \\
& \widehat{f}_{q}^{(r, i)}(-t)= \begin{cases}(-1)^{i} f_{q}^{(r, i)}(t) & \text { if } r \text { is odd } \\
(-1)^{i} \widehat{f}_{q}^{(r, i)}(t) & \text { if } r \text { is even }\end{cases}
\end{aligned}
$$

It is clear to see the following.

Proposition 1. For $n \geq 0$ and $i, j=0,1, \ldots, r-1$,

$$
\begin{aligned}
& c_{n}^{(r, i)}\left(p, \zeta_{r}^{j} q\right)=\zeta_{r}^{i j} c_{n}^{(r, i)}(p, q), \\
& \widehat{c}_{n}^{(r, i)}\left(p, \zeta_{r}^{j} q\right)=\zeta_{r}^{i j} \widehat{c}_{n}^{(r, i)}(p, q),
\end{aligned}
$$

where $\zeta$ is the primitive $r$-th root of unity.

In the next section, we show several properties of bivariate Cauchy polynomials with higher level. In particular, Theorem 1 entails fundamental recurrence formulas. By using these formulas, we give determinant expressions of bivariate Cauchy polynomials with higher levels. In special cases, we can get determinant expressions of the classical Cauchy polynomials and numbers.

\section{Basic Properties}

In this section, we show several properties of bivariate Cauchy polynomials with higher levels. We introduce the auxiliary polynomials $G_{n}^{(r, i)}(p, q)$ and $\widehat{G}_{n}^{(r, i)}(p, q)$ as

$$
\begin{aligned}
& \frac{f_{q}^{(r, i)}(t)}{(1+t)^{p}}=\sum_{n=0}^{\infty} G_{n}^{(r, i)}(p, q) \frac{t^{n}}{n !}, \\
& \frac{\widehat{f}_{q}^{(r, i)}(t)}{(1+t)^{p}}=\sum_{n=0}^{\infty} \widehat{G}_{n}^{(r, i)}(p, q) \frac{t^{n}}{n !} .
\end{aligned}
$$

respectively. 
Proposition 2. For $n \geq 0$ and $i=0,1, \ldots, r-1$,

$$
\begin{aligned}
& G_{n}^{(r, i)}(p, q)=\sum_{k=0}^{\left\lfloor\frac{n-i}{r}\right\rfloor}(-1)^{k}\left(\begin{array}{c}
n \\
r k+i
\end{array}\right)(-p)_{n-r k-i} q^{r k+i}, \\
& \widehat{G}_{n}^{(r, i)}(p, q)=\sum_{k=0}^{\left\lfloor\frac{n-i}{r}\right\rfloor}\left(\begin{array}{c}
n \\
r k+i
\end{array}\right)(-p)_{n-r k-i} q^{r k+i},
\end{aligned}
$$

where $(x)_{\ell}=x(x-1) \cdots(x-\ell+1)(\ell \geq 1)$ denotes the falling factorial with $(x)_{0}=1$.

Proof. From the definition in (9), we have

$$
\begin{aligned}
\sum_{n=0}^{\infty} G_{n}^{(r, i)}(p, q) \frac{t^{n}}{n !} & =\left(\sum_{l=0}^{\infty} \frac{(-p)_{l} t^{l}}{l !}\right)\left(\sum_{k=0}^{\infty}(-1)^{k} \frac{(q t)^{r k+i}}{(r k+i) !}\right) \\
& =\sum_{n=0}^{\infty} \sum_{k=0}^{\left\lfloor\frac{n-i}{r}\right\rfloor}(-1)^{k}\left(\begin{array}{c}
n \\
r k+i
\end{array}\right)(-p)_{n-r k-i} q^{r k+i} \frac{t^{n}}{n !}
\end{aligned}
$$

The identity (11) is obtained by comparing the coefficients on both sides. The identity (12) is similarly proved.

$c_{n}^{(r, i)}(p, q)$ (respectively, $\left.\widehat{c}_{n}^{(r, i)}(p, q)\right)$ can be written in terms of $G_{n}^{(r, i)}(p, q)$ (respectively, $\left.\widehat{G}_{n}^{(r, i)}(p, q)\right)$.

Proposition 3. For $n \geq 0$ and $i=0,1, \ldots, r-1$,

$$
\begin{aligned}
& c_{n}^{(r, i)}(p, q)=\sum_{k=0}^{n}\left(\begin{array}{l}
n \\
k
\end{array}\right) c_{n-k} G_{k}^{(r, i)}(p, q), \\
& \widehat{c}_{n}^{(r, i)}(p, q)=\sum_{k=0}^{n}\left(\begin{array}{l}
n \\
k
\end{array}\right) c_{n-k} \widehat{G}_{k}^{(r, i)}(p, q) .
\end{aligned}
$$

Proof.

$$
\begin{aligned}
\sum_{n=0}^{\infty} \widehat{c}_{n}^{(r, i)}(p, q) \frac{t^{n}}{n !} & =\frac{t}{\log (1+t)} \cdot \frac{\widehat{f}_{q}^{(r, i)}(t)}{(1+t)^{p}} \\
& =\left(\sum_{l=0}^{\infty} c_{l} \frac{t^{l}}{l !}\right)\left(\sum_{k=0}^{\infty} G_{k}^{(r, i)}(p, q) \frac{t^{k}}{k !}\right) \\
& =\sum_{n=0}^{\infty} \sum_{k=0}^{n}\left(\begin{array}{l}
n \\
k
\end{array}\right) c_{n-k} G_{k}^{(r, i)}(p, q) \frac{t^{n}}{n !}
\end{aligned}
$$

The identity (14) is obtained by comparing the coefficients on both sides. The identity (13) is similarly proved.

Contrary to Proposition $3, G_{n}^{(r, i)}(p, q)$ (respectively, $\widehat{G}_{n}^{(r, i)}(p, q)$ ) can be written in terms of $c_{n}^{(r, i)}(p, q)$ (respectively, $\left.\widehat{c}_{n}^{(r, i)}(p, q)\right)$.

Proposition 4. For $n \geq 0$ and $i=0,1, \ldots, r-1$,

$$
G_{n}^{(r, i)}(p, q)=\sum_{k=0}^{n} \frac{(-1)^{n-k} n !}{(n-k+1) k !} c_{k}^{(r, i)}(p, q),
$$




$$
\widehat{G}_{n}^{(r, i)}(p, q)=\sum_{k=0}^{n} \frac{(-1)^{n-k} n !}{(n-k+1) k !} \widehat{c}_{k}^{(r, i)}(p, q)
$$

\section{Proof.}

$$
\begin{aligned}
\sum_{n=0}^{\infty} G_{n}^{(r, i)}(p, q) \frac{t^{n}}{n !} & =\frac{\log (1+t)}{t} \sum_{k=0}^{\infty} c_{k}^{(r, i)}(p, q) \frac{t^{k}}{k !} \\
& =\left(\sum_{l=0}^{\infty} \frac{(-1)^{l} t^{l}}{l+1}\right)\left(\sum_{k=0}^{\infty} c_{k}^{(r, i)}(p, q) \frac{t^{k}}{k !}\right) \\
& =\sum_{n=0}^{\infty} \sum_{k=0}^{n} \frac{(-1)^{n-k} n !}{(n-k+1) k !} c_{k}^{(r, i)}(p, q) \frac{t^{n}}{n !} .
\end{aligned}
$$

The identity (15) is obtained by comparing the coefficients on both sides. The identity (16) is similarly proved.

We have a summation formula for $c_{n}^{(r, i)}(p, q)$ (respectively, $\left.\widehat{c}_{n}^{(r, i)}(p, q)\right)$.

Theorem 1. For $n \geq 0$ and $i=0,1, \ldots, r-1$,

$$
\begin{aligned}
& \sum_{k=0}^{n}\left(\begin{array}{c}
n+1 \\
k
\end{array}\right) c_{k}^{(r, i)}(p, q) \frac{d}{d p}(p)_{n-k+1} \\
& = \begin{cases}-(-1)^{(n-i) / r}(n+1) q^{n} & \text { if } n \equiv i \quad(\bmod r) \\
0 & \text { otherwise }\end{cases} \\
& \sum_{k=0}^{n}\left(\begin{array}{c}
n+1 \\
k
\end{array}\right) \widehat{c}_{k}^{(r, i)}(p, q) \frac{d}{d p}(p)_{n-k+1} \\
& = \begin{cases}-(n+1) q^{n} & \text { if } n \equiv i \quad(\bmod r) \\
0 & \text { otherwise. }\end{cases}
\end{aligned}
$$

When $p=0$ in Theorem 1 , by

$$
\left.\frac{d}{d p}(p)_{n-k+1}\right|_{p=0}=(-1)^{n-k}(n-k) !
$$

we have simpler recurrence relations.

Corollary 1. For $n \geq 0$ and $i=0,1, \ldots, r-1$,

$$
\begin{aligned}
& \sum_{k=0}^{n} \frac{(-1)^{n-k} c_{k}^{(r, i)}(p, q)}{(n-k+1) k !}= \begin{cases}-(-1)^{(n-i) / r} \frac{q^{n}}{n !} & \text { if } n \equiv i \quad(\bmod r) \\
0 & \text { otherwise }\end{cases} \\
& \sum_{k=0}^{n} \frac{(-1)^{n-k} \widehat{c}_{k}^{(r, i)}(p, q)}{(n-k+1) k !}= \begin{cases}-\frac{q^{n}}{n !} & \text { if } n \equiv i \quad(\bmod r) \\
0 & \text { otherwise. }\end{cases}
\end{aligned}
$$

Proof of Theorem 1. From the definition in (1) and the proof of Proposition 4, we have

$$
f_{q}^{(r, i)}(t)=(1+t)^{p} \cdot \frac{\log (1+t)}{t} \sum_{n=0}^{\infty} c_{n}^{(r, i)}(p, q) \frac{t^{n}}{n !}
$$




$$
\begin{aligned}
= & \left(\sum_{m=0}^{\infty} \frac{(p)_{m}}{m !} t^{m}\right)\left(\sum_{l=0}^{\infty} \sum_{k=0}^{l} \frac{(-1)^{l-k+1}}{(l-k+1) k !} c_{k}^{(r, i)}(p, q) t^{l}\right) \\
= & \sum_{n=0}^{\infty}\left(\sum_{l=0}^{n} \frac{(p)_{n-l}}{(n-l) !} \sum_{k=0}^{l} \frac{(-1)^{l-k+1} n !}{(l-k+1) k !} c_{k}^{(r, i)}(p, q)\right) \frac{t^{n}}{n !} \\
= & \sum_{n=0}^{\infty}\left(\sum_{k=0}^{n} c_{k}^{(r, i)}(p, q) \sum_{l=k}^{n}\left(\begin{array}{l}
n \\
l
\end{array}\right)\left(\begin{array}{l}
l \\
k
\end{array}\right) \frac{(-p)^{n-l}}{(l-k+1)}\right) \frac{t^{n}}{n !} \\
= & \sum_{n=0}^{\infty}\left(\sum_{k=0}^{n} \frac{(-1)^{k-1} c_{k}^{(r, i)}(p, q)}{k !} \sum_{l=k}^{n} \frac{(-1)^{l} n !(p)_{n-l}}{(l-k+1)(n-l) !}\right) \frac{t^{n}}{n !} \\
= & \sum_{n=0}^{\infty}\left(\sum_{k=0}^{n} \frac{(-1)^{k-1} c_{k}^{(r, i)}(p, q)}{k !}(-1)^{n} n ! \sum_{j=0}^{n-k} \frac{(-1)^{j}(p)_{j}}{(n-k-j+1) j !}\right) \frac{t^{n}}{n !} .
\end{aligned}
$$

Since

$$
\begin{aligned}
\sum_{j=0}^{n-k} \frac{(-1)^{j}(p)_{j}}{(n-k-j+1) j !} & =\frac{1}{(n-k+1) !} \sum_{l=0}^{n-k}(-1)^{l}(l+1)\left[\begin{array}{c}
n-k+1 \\
l+1
\end{array}\right] p^{l} \\
& =\frac{1}{(n-k+1) !} \frac{d}{d p}(-1)^{n-k}(p)_{n-k+1},
\end{aligned}
$$

where $\left[\begin{array}{l}n \\ k\end{array}\right]$ denotes the (unsigned) Stirling numbers of the first kind as in

$$
(x)_{n}=\sum_{k=0}^{n}(-1)^{n-k}\left[\begin{array}{l}
n \\
k
\end{array}\right] x^{k},
$$

comparing the coefficients with

$$
f_{q}^{(r, i)}(t)=\sum_{n=0}^{\infty}(-1)^{n} \frac{(q t)^{r n+i}}{(r n+i) !}
$$

in (2), we get the identity (17). The identity (18) is similarly proved.

From Theorem 1, we have the recurrence relations

$$
\begin{aligned}
c_{n}^{(r, i)}(p, q)= & -n ! \sum_{k=0}^{n-1} \frac{c_{k}^{(r, i)}(p, q)}{k !} \frac{d}{d p} \frac{(p)_{n-k+1}}{(n-k+1) !} \\
& - \begin{cases}(-1)^{(n-i) / r} q^{n} & \text { if } n \equiv i \quad(\bmod r) ; \\
0 & \text { otherwise, }\end{cases} \\
\widehat{c}_{n}^{(r, i)}(p, q)= & -n ! \sum_{k=0}^{n-1} \frac{\widehat{c}_{k}^{(r, i)}(p, q)}{k !} \frac{d}{d p} \frac{(p)_{n-k+1}}{(n-k+1) !} \\
& -\left\{\begin{array}{lll}
q^{n} & \text { if } n \equiv i \quad(\bmod r) ; \\
0 & \text { otherwise }
\end{array}\right.
\end{aligned}
$$

with

$$
c_{0}^{(r, i)}(p, q)=\widehat{c}_{0}^{(r, i)}(p, q)= \begin{cases}1 & \text { if } i=0 \\ 0 & \text { if } i=1,2 .\end{cases}
$$

By using recursions, we can get the exact values of $c_{n}^{(r, i)}(p, q)$ and $\widehat{c}_{n}^{(r, i)}(p, q)$ for small $n$. We list some initial values in the Appendix A. 
Theorem 2. For $n \geq 0$ and $i=0,1, \ldots, r-1$,

$$
\begin{aligned}
& c_{n}^{(r, i)}(p+1, q)-c_{n}^{(r, i)}(p, q)=-n c_{n-1}^{(r, i)}(p+1, q), \\
& \widehat{c}_{n}^{(r, i)}(p+1, q)-\widehat{c}_{n}^{(r, i)}(p, q)=-n \widehat{c}_{n-1}^{(r, i)}(p+1, q) .
\end{aligned}
$$

Proof.

$$
\begin{aligned}
& \sum_{n=0}^{\infty} c_{n}^{(r, i)}(p+1, q) \frac{t^{n}}{n !}-\sum_{n=0}^{\infty} c_{n}^{(r, i)}(p, q) \frac{t^{n}}{n !} \\
& =\frac{t f_{q}^{(r, i)}(t)}{(1+t)^{p+1} \log (1+t)}-\frac{t f_{q}^{(r, i)}(t)}{(1+t)^{p} \log (1+t)} \\
& =-\frac{t^{2} f_{q}^{(r, i)}(t)}{(1+t)^{p+1} \log (1+t)} \\
& =-t \sum_{n=0}^{\infty} c_{n}^{(r, i)}(p+1, q) \frac{t^{n}}{n !} \\
& =-\sum_{n=0}^{\infty} n c_{n-1}^{(r, i)}(p+1, q) \frac{t^{n}}{n !} .
\end{aligned}
$$

The identity (21) is obtained by comparing the coefficients on both sides. The identity (22) is similarly proved.

Theorem 3. For $n \geq 0$ and $i=0,1, \ldots, r-1$,

$$
\begin{aligned}
& c_{n}^{(r, i)}(p+s, q)=\sum_{k=0}^{n}\left(\begin{array}{l}
n \\
k
\end{array}\right) c_{k}^{(r, i)}(p, q)(-s)_{n-k}, \\
& \widehat{c}_{n}^{(r, i)}(p+s, q)=\sum_{k=0}^{n}\left(\begin{array}{l}
n \\
k
\end{array}\right) \widehat{c}_{k}^{(r, i)}(p, q)(-s)_{n-k} .
\end{aligned}
$$

\section{Proof.}

$$
\begin{aligned}
\sum_{n=0}^{\infty} c_{n}^{(r, i)}(p+s, q) \frac{t^{n}}{n !} & =\frac{t f_{q}^{(r, i)}(t)}{(1+t)^{p+s} \log (1+t)} \\
& =\left(\sum_{n=0}^{\infty} c_{n}^{(r, i)}(p, q) \frac{t^{n}}{n !}\right)\left(\sum_{l=0}^{\infty} \frac{(-s)_{l} t^{l}}{l !}\right) \\
& =\sum_{n=0}^{\infty} \sum_{k=0}^{n}\left(\begin{array}{l}
n \\
k
\end{array}\right) c_{k}^{(r, i)}(p, q)(-s)_{n-k} \frac{t^{n}}{n !} .
\end{aligned}
$$

The identity (23) is obtained by comparing the coefficients on both sides. The identity (24) is similarly proved.

Theorem 4. For $n \geq 0$ and $i=0,1, \ldots, r-1$,

$$
\begin{aligned}
& \sum_{k=0}^{n} \frac{c_{k}^{(r, i)}(p, q)}{k !}=-\frac{c_{n}^{(r, i)}(p+1, q)}{n !}, \\
& \sum_{k=0}^{n} \frac{\widehat{c}_{k}^{(r, i)}(p, q)}{k !}=-\frac{\widehat{c}_{n}^{(r, i)}(p+1, q)}{n !} .
\end{aligned}
$$


Proof. By using Theorem 3 (23) with $r=1$ and $r=0$,

$$
\begin{aligned}
c_{n+1}^{(r, i)}(p+1, q)-c_{n+1}^{(r, i)}(p, q) & =\sum_{k=0}^{n}\left(\begin{array}{c}
n+1 \\
k
\end{array}\right) c_{k}^{(r, i)}(p, q) \cdot(-1)^{n-k+1}(n-k+1) ! \\
& =(n+1) ! \sum_{k=0}^{n}(-1)^{n-k+1} \frac{c_{k}^{(r, i)}(p, q)}{k !} .
\end{aligned}
$$

Together with Theorem 2 (21), we get the identity (25). The identity (26) is similarly proved.

Theorem 5. For $n \geq 1$,

$$
\begin{aligned}
\frac{\partial}{\partial p} c_{n}^{(r, i)}(p, q) & =\sum_{k=0}^{n-1}(-1)^{n-k-1} \frac{c_{k}^{(r, i)}(p, q)}{(n-k) k !} \quad(i=0,1, \ldots, r-1), \\
\frac{\partial}{\partial q} c_{n}^{(r, i)}(p, q) & = \begin{cases}-n c_{n-1}^{(r, r-1)}(p, q) & \text { if } i=0 ; \\
n c_{n-1}^{(r, i-1)}(p, q) & \text { if } i=1,2, \ldots, r-1,\end{cases} \\
\frac{\partial}{\partial p} \widehat{c}_{n}^{(r, i)}(p, q) & =\sum_{k=0}^{n-1}(-1)^{n-k-1} \frac{\widehat{c}_{k}^{(r, i)}(p, q)}{(n-k) k !} \quad(i=0,1, \ldots, r-1), \\
\frac{\partial}{\partial q} \widehat{c}_{n}^{(r, i)}(p, q) & = \begin{cases}n \widehat{c}_{n-1}^{(r, r-1)}(p, q) & \text { if } i=0 ; \\
n \widehat{c}_{n-1}^{(r, i-1)}(p, q) & \text { if } i=1,2, \ldots, r-1 .\end{cases}
\end{aligned}
$$

\section{Proof.}

$$
\begin{aligned}
\sum_{n=1}^{\infty} \frac{\partial}{\partial p} c_{n}^{(r, i)}(p, q) \frac{t^{n}}{n !} & =-\frac{t f_{q}^{(r, i)}(t)}{(1+t)^{p}} \\
& =\left(\sum_{m=1}^{\infty}(-1)^{m-1} \frac{t^{m}}{m !}\right)\left(\sum_{n=0}^{\infty} c_{n}^{(r, i)}(p, q) \frac{t^{n}}{n !}\right) \\
& =\sum_{n=0}^{\infty} \sum_{k=0}^{n-1}(-1)^{n-k-1} \frac{c_{k}^{(r, i)}(p, q)}{(n-k) k !} \frac{t^{n}}{n !},
\end{aligned}
$$

yielding (27). Since

$$
\begin{aligned}
\frac{\partial}{\partial q} f_{q}^{(r, 0)}(t) & =t \sum_{n=1}^{\infty} \frac{(-1)^{n}(q t)^{r n-1}}{(r n-1) !} \\
& =-t f_{q}^{(r, r-1)}(t)
\end{aligned}
$$

and

$$
\frac{\partial}{\partial q} f_{q}^{(r, i)}(t)=t f_{q}^{(r, i-1)}(t) \quad(i=1,2, \ldots, r-1),
$$

we get (28). The identities (29) and (30) are similarly proved.

\section{Determinants}

Bivariate Cauchy polynomials $c_{n}^{(r, 0)}(p, q)$ with a higher level and their complementary numbers $\widehat{c}_{n}^{(r, 0)}(p, q)$ have determinant expressions. 
Theorem 6. For $n \geq i+1$,

$$
c_{n}^{(r, i)}(p, q)=\frac{q^{i} n !}{i !}\left|\begin{array}{ccccc}
r_{p}(2) & 1 & 0 & \cdots & 0 \\
r_{p}(3) & r_{p}(2) & 1 & & \vdots \\
\vdots & \vdots & \ddots & \ddots & 0 \\
r_{p}(n-i) & r_{p}(n-i-1) & \cdots & r_{p}(2) & 1 \\
r_{p}^{*}(n-i+1) & r_{p}^{*}(n-i) & \cdots & r_{p}^{*}(3) & r_{p}^{*}(2)
\end{array}\right|
$$

and

$$
\widehat{c}_{n}^{(r, 0)}(p, q)=\frac{q^{i} n !}{i !}\left|\begin{array}{ccccc}
r_{p}(2) & 1 & 0 & \cdots & 0 \\
r_{p}(3) & r_{p}(2) & 1 & & \vdots \\
\vdots & \vdots & \ddots & \ddots & 0 \\
r_{p}(n-i) & r_{p}(n-i-1) & \cdots & r_{p}(2) & 1 \\
\widehat{r}_{p}(n-i+1) & \widehat{r}_{p}(n-i) & \cdots & \widehat{r}_{p}(3) & \widehat{r}_{p}(2)
\end{array}\right|,
$$

where

$$
\begin{aligned}
r_{p}(n) & =\frac{d}{d p} \frac{(-1)^{n-1}(p)_{n}}{n !} \\
& =\frac{1}{n !} \sum_{\ell=0}^{n-1}(-1)^{\ell}(\ell+1)\left[\begin{array}{c}
n \\
\ell+1
\end{array}\right] p^{\ell}
\end{aligned}
$$

with

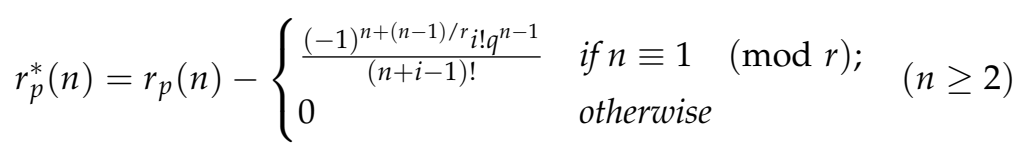

and

$$
\widehat{r}_{p}(n)=r_{p}(n)+\left\{\begin{array}{ll}
\frac{(-1)^{n} i ! q^{n-1}}{(n+i-1) !} & \text { if } n \equiv 1 \quad(\bmod r) ; \\
0 & \text { otherwise }
\end{array} \quad(n \geq 2) .\right.
$$

Proof. From Theorem 1 with the recurrence relation in (19), putting

$$
\gamma_{n}:=\frac{i !}{q^{i}} \frac{c_{n}^{(r, i)}(p, q)}{n !}
$$

we have

$$
\gamma_{n}=\sum_{k=i}^{n-1}(-1)^{n-k+1} r_{p}(n-k+1) \gamma_{k}- \begin{cases}\frac{(-1)^{(n-i) / r} i ! q^{n-i}}{n !} & \text { if } n \equiv i \quad(\bmod r) \\ 0 & \text { otherwise }\end{cases}
$$

Notice that $\gamma_{0}=\cdots=\gamma_{i-1}=0$ and $\gamma_{i}=1$ since $c_{0}^{(r, i)}(p, q)=\cdots=c_{i-1}^{(r, i)}(p, q)=0$ and $c_{i}^{(r, i)}(p, q)=q^{i}$. By induction, we shall prove that

$$
\gamma_{n}=\left|\begin{array}{ccccc}
r_{p}(2) & 1 & 0 & \cdots & 0 \\
r_{p}(3) & r_{p}(2) & 1 & & \vdots \\
\vdots & \vdots & \ddots & \ddots & 0 \\
r_{p}(n-i) & r_{p}(n-i-1) & \cdots & r_{p}(2) & 1 \\
\widehat{r}_{p}(n-i+1) & \widehat{r}_{p}(n-i) & \cdots & \widehat{r}_{p}(3) & \widehat{r}_{p}(2)
\end{array}\right|
$$


For $n=i+1$, by the recurrence relation (31), we get

$$
\gamma_{i+1}=r_{p}(2)=\left|r_{p}(2)\right| .
$$

Assume that the determinant expression of (32) is valid up to $n-1$. Then, by expanding the right-hand-side of (32) along the first row repeatedly, we have

$$
\begin{aligned}
& r_{p}(2) \gamma_{n-1}-\left|\begin{array}{ccccc}
r_{p}(3) & 1 & 0 & \cdots & 0 \\
r_{p}(4) & r_{p}(2) & 1 & & \vdots \\
\vdots & \vdots & \ddots & \ddots & 0 \\
r_{p}(n-i) & r_{p}(n-i-2) & \cdots & r_{p}(2) & 1 \\
r_{p}^{*}(n-i+1) & r_{p}^{*}(n-i-1) & \cdots & r_{p}^{*}(3) & r_{p}^{*}(2)
\end{array}\right| \\
&=r_{p}(2) \gamma_{n-1}-r_{p}(3) \gamma_{n-2}+\cdots+(-1)^{n-i-1} r_{p}(n-1) \gamma_{i+2} \\
&+(-1)^{n-i}\left|\begin{array}{cc}
r_{p}(n-i) & 1 \\
r_{p}^{*}(n-i+1) & r_{p}^{*}(2)
\end{array}\right| \\
&= r_{p}(2) \gamma_{n-1}-r_{p}(3) \gamma_{n-2}+\cdots+(-1)^{n-i-1} r_{p}(n-1) \gamma_{i+2} \\
&+(-1)^{n-i} r_{p}(n) \gamma_{i+1}+(-1)^{n-i+1} r_{p}^{*}(n-i+1) \gamma_{i} \\
&= \gamma_{n} .
\end{aligned}
$$

The last identity is entailed from the recurrence relation (31) and

$$
r_{p}^{*}(n-i+1)=r_{p}(n-i+1)+(-1)^{n-i+1} \frac{(-1)^{(n-i) / r} q^{n-i}}{n !}
$$

for $n \equiv i(\bmod r)$. By putting $m=n-i+1$, we find that

$$
r_{p}^{*}(m)=r_{p}(m)-\frac{(-1)^{m+(m-1) / r} i ! q^{m-1}}{(m+i-1) !}
$$

for $m \equiv 1(\bmod r)$. In addition,

$$
\begin{aligned}
\sum_{j=0}^{n-k} \frac{(-1)^{j}(p)_{j}}{(n-k-j+1) j !} & =\frac{1}{(n-k+1) !} \sum_{l=0}^{n-k}(-1)^{l}(l+1)\left[\begin{array}{c}
n-k+1 \\
l+1
\end{array}\right] p^{l} \\
& =\frac{1}{(n-k+1) !} \frac{d}{d p}(-1)^{n-k}(p)_{n-k+1} .
\end{aligned}
$$

Another identity can be yielded similarly from the recurrence relation

$$
\widehat{\gamma}_{n}=\sum_{k=i}^{n-1}(-1)^{n-k+1} r_{p}(n-k+1) \widehat{\gamma}_{k}+ \begin{cases}\frac{i ! q^{n-i}}{n !} & \text { if } n \equiv i \quad(\bmod r) ; \\ 0 & \text { otherwise }\end{cases}
$$

with

$$
\widehat{\gamma}_{n}:=\frac{i !}{q^{i}} \frac{\widehat{c}_{n}^{(r, i)}(p, q)}{n !}
$$


When $q=i=0$ in Theorem 6, a simpler determinant expression of Cauchy polynomials $c_{n}(p):=c_{n}^{(r, 0)}(p, 0)=\widehat{c}_{n}^{(r, 0)}(p, 0)$ is given. For simplification of determinant expressions, we use the Jordan matrix

$$
J=\left(\begin{array}{ccccc}
0 & 0 & \cdots & 0 & 0 \\
1 & 0 & \cdots & 0 & 0 \\
0 & 1 & \cdots & 0 & 0 \\
\vdots & \vdots & \cdots & \vdots & \vdots \\
0 & 0 & \cdots & 1 & 0
\end{array}\right)
$$

$J^{0}$ is an identity matrix and $J^{T}$ is the transpose matrix of $J$.

Corollary 2. For $n \geq 1$,

$$
c_{n}(p)=\widehat{c}_{n}(p)=n !\left|J^{T}+\sum_{k=1}^{n} r_{p}(k+1) J^{k-1}\right| .
$$

Remark 1. When $p=0$ in Corollary 2, by

$$
r_{0}(n)=\left.\frac{d}{d p} \frac{(-1)^{n-1}(p)_{n}}{n !}\right|_{p=0}=\frac{1}{n},
$$

we have a determinant expression of Cauchy numbers $c_{n}$ as

$$
c_{n}=n !\left|J^{T}+\sum_{k=1}^{n} \frac{1}{k+1} J^{k-1}\right|
$$

(p. $50[30])$.

We need the following equivalent relations (see, e.g., [31]).

Lemma 1.

$$
\begin{gathered}
\sum_{k=0}^{n}(-1)^{n-k} x_{n-k} z_{k}=0 \quad \text { with } \quad x_{0}=z_{0}=1 \\
\Longleftrightarrow \quad x_{n}=\left|J^{T}+\sum_{k=1}^{n} z_{k} J^{k-1}\right| \quad \Longleftrightarrow \quad z_{n}=\left|J^{T}+\sum_{k=1}^{n} x_{k} J^{k-1}\right| .
\end{gathered}
$$

By using Lemma 1 again, we have the inversion relation of Corollary 2.

Corollary 3. For $n \geq 1$,

$$
r_{p}(n+1)=\left|J^{T}+\sum_{k=1}^{n} \frac{c_{k}(p)}{k !} J^{k-1}\right|
$$

We shall use Trudi's formula to obtain different explicit expressions and inversion relations for the numbers $c_{n}^{(r, i)}(p, 0)$.

Lemma 2. For $n \geq 1$, we have

$$
\left|a_{0} J^{T}+\sum_{k=1}^{n} a_{k} J^{k-1}\right|=\sum_{t_{1}+2 t_{2}+\cdots+n t_{n}=n}\left(\begin{array}{c}
t_{1}+\cdots+t_{n} \\
t_{1}, \ldots, t_{n}
\end{array}\right)\left(-a_{0}\right)^{n-t_{1}-\cdots-t_{n}} a_{1}^{t_{1}} a_{2}^{t_{2}} \cdots a_{n}^{t_{n}},
$$


where $\left(\begin{array}{c}t_{1}+\cdots+t_{n} \\ t_{1}, \ldots, t_{n}\end{array}\right)=\frac{\left(t_{1}+\cdots+t_{n}\right) !}{t_{1} ! \cdots t_{n} !}$ are the multinomial coefficients.

This relation is known as Trudi's formula [32] (Volume 3, p. 214), [33] and the case $a_{0}=1$ of this formula is known as Brioschi's formula [34], (Volume 3, pp. 208-209) [32].

By Corollaries 2 and 3 with Lemma 2, we get different expressions of $c_{n}(p)$ and $r_{p}(n)$.

Corollary 4. For $n \geq 1$,

$$
\begin{aligned}
c_{n}(p)= & \sum_{t_{1}+2 t_{2}+\cdots+n t_{n}=n}\left(\begin{array}{c}
t_{1}+\cdots+t_{n} \\
t_{1}, \ldots, t_{n}
\end{array}\right) \\
& \times(-1)^{n-t_{1}-\cdots-t_{n}}\left(r_{p}(2)\right)^{t_{1}}\left(r_{p}(3)\right)^{t_{2}} \cdots\left(r_{p}(n+1)\right)^{t_{n}}, \\
r_{p}(n+1)= & \sum_{t_{1}+2 t_{2}+\cdots+n t_{n}=n}\left(\begin{array}{c}
t_{1}+\cdots+t_{n} \\
t_{1}, \ldots, t_{n}
\end{array}\right) \\
& \times(-1)^{n-t_{1}-\cdots-t_{n}}\left(c_{1}(p)\right)^{t_{1}}\left(\frac{c_{2}(p)}{2 !}\right)^{t_{2}} \cdots\left(\frac{c_{n}(p)}{n !}\right)^{t_{n}} .
\end{aligned}
$$

\section{Discussion}

Cauchy numbers and polynomials have been often considered and studied in relation to Bernoulli polynomials and numbers. There are many generalizations of Cauchy polynomials. In particular, poly-Cauchy numbers are analogues of poly-Bernoulli numbers, and hypergeometric Cauchy numbers are analogues of hypergeometric Bernoulli numbers. Recently, parametric types of Cauchy polynomials with level 2 (bivariate) and level 3 (trivariate) are introduced and studied as analogues of parametric types of Bernoulli polynomials with level 2 and level 3, respectively. In this paper, such a concept is totally generalized as Cauchy polynomials with a higher level. We give their characteristic and combinatorial properties, including determinant expressions.

Funding: This research received no external funding.

Data Availability Statement: Not applicable.

Acknowledgments: The author thanks the anonymous referees for careful reading of the manuscript and useful suggestions.

Conflicts of Interest: The author declares no conflict of interest.

\section{Appendix A}

By using recursions in (19) and (20) or the determinant expressions in Theorem 6, we can get the exact values of $c_{n}^{(r, i)}(p, q)$ and $\widehat{c}_{n}^{(r, i)}(p, q)$ for small $n$. We list some initial values.

$$
\begin{aligned}
& c_{0}^{(3,0)}(p, q)=1 \\
& c_{1}^{(3,0)}(p, q)=-p+\frac{1}{2}, \\
& c_{2}^{(3,0)}(p, q)=p^{2}-\frac{1}{6}, \\
& c_{3}^{(3,0)}(p, q)=-p^{3}-\frac{3 p^{2}}{2}-q^{3}+\frac{1}{4}, \\
& c_{4}^{(3,0)}(p, q)=p^{4}+4 p^{3}+4 p^{2}+4 q^{3} p-2 q^{3}-\frac{19}{30}, \\
& c_{5}^{(3,0)}(p, q)=-p^{5}-\frac{15 p^{4}}{2}-\frac{55 p^{3}}{3}-5\left(2 q^{3}+3\right) p^{2}+\frac{5 q^{3}}{3}+\frac{9}{4}, \\
& c_{6}^{(3,0)}(p, q)=p^{6}+12 p^{5}+\frac{105 p^{4}}{2}+20\left(q^{3}+5\right) p^{3}+6\left(5 q^{3}+12\right) p^{2}+q^{6}-5 q^{3}-\frac{863}{84} .
\end{aligned}
$$




$$
\begin{aligned}
& c_{0}^{(3,1)}(p, q)=0, \\
& c_{1}^{(3,1)}(p, q)=q, \\
& c_{2}^{(3,1)}(p, q)=-2 q p+q, \\
& c_{3}^{(3,1)}(p, q)=3 q p^{2}-\frac{q}{2}, \\
& c_{4}^{(3,1)}(p, q)=-4 q p^{3}+6 q p^{2}-q^{4}+q, \\
& c_{5}^{(3,1)}(p, q)=5 q p^{4}+20 q p^{3}+20 q p^{2}+5 q^{4} p-\frac{5 q^{4}}{2}-\frac{19 q}{6}, \\
& c_{6}^{(3,1)}(p, q)=-6 q p^{5}-45 q p^{4}-110 q p^{3}-15 q\left(q^{3}+6\right) p^{2}+\frac{5 q^{4}}{2}+\frac{27 q}{2} . \\
& c_{0}^{(3,2)}(p, q)=0, \\
& c_{1}^{(3,2)}(p, q)=0, \\
& c_{2}^{(3,2)}(p, q)=q^{2}, \\
& c_{3}^{(3,2)}(p, q)=-3 q^{2} p+\frac{3 q^{2}}{2}, \\
& c_{4}^{(3,2)}(p, q)=6 q^{2} p^{2}-q^{2}, \\
& c_{5}^{(3,2)}(p, q)=-10 q^{2} p^{3}-15 q^{2} p^{2}-q^{5}+\frac{5 q^{2}}{2}, \\
& c_{6}^{(3,2)}(p, q)=15 q^{2} p^{4}+60 q^{2} p^{3}+60 q^{2} p^{2}+6 q^{5} p-3 q^{5}-\frac{19 q^{2}}{2} .
\end{aligned}
$$

$$
\begin{aligned}
& \widehat{c}_{0}^{(3,0)}(p, q)=1 \\
& \widehat{c}_{1}^{(3,0)}(p, q)=-p+\frac{1}{2} \\
& \widehat{c}_{2}^{(3,0)}(p, q)=p^{2}-\frac{1}{6} \\
& \widehat{c}_{3}^{(3,0)}(p, q)=-p^{3}-\frac{3 p^{2}}{2}+q^{3}+\frac{1}{4}, \\
& \widehat{c}_{4}^{(3,0)}(p, q)=p^{4}+4 p^{3}+4 p^{2}-4 q^{3} p+2 q^{3}-\frac{19}{30}, \\
& \widehat{c}_{5}^{(3,0)}(p, q)=-p^{5}-\frac{15 p^{4}}{2}-\frac{55 p^{3}}{3}+5\left(2 q^{3}-3\right) p^{2}-\frac{5 q^{3}}{3}+\frac{9}{4}, \\
& \widehat{c}_{6}^{(3,0)}(p, q)=p^{6}+12 p^{5}+\frac{105 p^{4}}{2}-20\left(q^{3}-5\right) p^{3}-6\left(5 q^{3}-12\right) p^{2}+q^{6}+5 q^{3}-\frac{863}{84} .
\end{aligned}
$$

$$
\begin{aligned}
& \widehat{c}_{0}^{(3,1)}(p, q)=0 \\
& \widehat{c}_{1}^{(3,1)}(p, q)=q, \\
& \widehat{c}_{2}^{(3,1)}(p, q)=-2 q p+q \\
& \widehat{c}_{3}^{(3,1)}(p, q)=3 q p^{2}-\frac{q}{2}, \\
& \widehat{c}_{4}^{(3,1)}(p, q)=-4 q p^{3}+6 q p^{2}+q^{4}+q, \\
& \widehat{c}_{5}^{(3,1)}(p, q)=5 q p^{4}+20 q p^{3}+20 q p^{2}-5 q^{4} p+\frac{5 q^{4}}{2}-\frac{19 q}{6}, \\
& \widehat{c}_{6}^{(3,1)}(p, q)=-6 q p^{5}-45 q p^{4}-110 q p^{3}+15 q\left(q^{3}-6\right) p^{2}-\frac{5 q^{4}}{2}+\frac{27 q}{2} .
\end{aligned}
$$




$$
\begin{aligned}
& \widehat{c}_{0}^{(3,2)}(p, q)=0 \\
& \widehat{c}_{1}^{(3,2)}(p, q)=0 \\
& \widehat{c}_{3}^{(3,2)}(p, q)=-3 q^{2} p+\frac{3 q^{2}}{2} \\
& \widehat{c}_{4}^{(3,2)}(p, q)=6 q^{2} p^{2}-q^{2} \\
& \widehat{c}_{5}^{(3,2)}(p, q)=-10 q^{2} p^{3}-15 q^{2} p^{2}+q^{5}+\frac{5 q^{2}}{2} \\
& \widehat{c}_{6}^{(3,2)}(p, q)=15 q^{2} p^{4}+60 q^{2} p^{3}+60 q^{2} p^{2}-6 q^{5} p+3 q^{5}-\frac{19 q^{2}}{2} .
\end{aligned}
$$

\section{References}

1. Cheon, G.-S.; Hwang, S.-G.; Lee, S.-G. Several polynomials associated with the harmonic numbers. Discrete Appl. Math. 2007, 155, 2573-2584. [CrossRef]

2. Comtet, L. Advanced Combinatorics; Riedel: Dordrech, The Netherlands; Boston, MA, USA, 1974.

3. Merlini, D.; Sprugnoli, R.; Verri, M.C. The Cauchy numbers. Discrete Math. 2006, 306, 1906-1920. [CrossRef]

4. Zhao, F.-Z. Sums of products of Cauchy numbers. Discrete Math. 2009, 309, 3830-3842. [CrossRef]

5. Agoh, T.; Dilcher, K. Recurrence relations for Nörlund numbers and Bernoulli numbers of the second kind. Fibonacci Quart. 2010, $48,4-12$.

6. Liu, G.-D.; Srivastava, H.M. Explicit formulas for the Norlund polynomials $B_{n}^{(x)}$ and $b_{n}^{(x)}$. Comput. Math. Appl. 2006, 51, 1377-1384. [CrossRef]

7. Nörlund, N.E. Vorlesungen über Differenzenrechnung; Springer: Berlin, Germany, 1924.

8. Howard, F.T. Explicit formulas for degenerate Bernoulli numbers. Discrete Math. 1996, 162, 175-185. [CrossRef]

9. Komatsu, T. Poly-Cauchy numbers. Kyushu J. Math. 2013, 67, 143-153. [CrossRef]

10. Wang, H.; Liu, G. An explicit formula for higher order Bernoulli polynomials of the second kind. Integers 2013, 13, 1-7.

11. Cenkci, M.; Howard, F.T. Notes on degenerate numbers. Discrete Math. 2007, 307, 2359-2375. [CrossRef]

12. Howard, F.T. Degenerate weighted Stirling numbers. Discrete Math. 1985, 57, 45-58. [CrossRef]

13. Komatsu, T. Hypergeometric Cauchy numbers. Int. J. Number Theory 2013, 9, 545-560. [CrossRef]

14. Carlitz, L. Degenerate Stirling, Bernoulli, and Eulerian numbers. Utilitas Math. 1979, 15, 51-88.

15. Kaneko, M. Poly-Bernoulli numbers. J. Théor. Nombres Bordx. 1997, 9, 199-206. [CrossRef]

16. Hassen, A.; Nguyen, H.D. Hypergeometric Bernoulli polynomials and Appell sequences. Int. J. Number Theory 2008, 4, 767-774. [CrossRef]

17. Hassen, A.; Nguyen, H.D. Hypergeometric zeta functions. Int. J. Number Theory 2010, 6, 99-126. [CrossRef]

18. Kamano, K. Sums of products of hypergeometric Bernoulli numbers. J. Number Theory 2010, 130, 2259-2271. [CrossRef]

19. Masjed-Jamei, M.; Beyki, M.R.; Koepf, W. Symbolic computation of some power-trigonometric series. J. Symb. Comput. 2017, 80, 273-284. [CrossRef]

20. Osler, T.J. An identity for simplifying certain generalized hypergeometric functions. Math. Comput. 1975, $29,888-893$.

21. Masjed-Jamei, M.; Beyki, M.R.; Koepf, W. An extension of the Euler-Maclaurin quadrature formula using a parametric type of Bernoulli polynomials. Bull. Sci. Math. 2019, 156, 102798. [CrossRef]

22. Komatsu, T. A parametric type of Bernoulli polynomials with level 3. Rev. R. Acad. Cienc. Exactas Fis. Nat. Ser. A Mat. RACSAM 2020, 114, Paper No. 151, 19p. [CrossRef]

23. Masjed-Jamei, M.; Beyki, M.R.; Koepf, W. A new type of Euler polynomials and numbers. Mediterr. J. Math. 2018, 15, 1-17. [CrossRef]

24. Srivastava, H.M.; Kízílateş, C. A parametric kind of the Fubini-type polynomials. Rev. R. Acad. Cienc. Exactas Fis. Nat. Ser. A Mat. RACSAM 2019, 113, 3253-3267. [CrossRef]

25. Srivastava, H.M.; Masjed-Jamei, M.; Beyki, M.R. Some new generalizations and applications of the Apostol-Bernoulli, ApostolEuler and Apostol-Genocchi polynomials. Rocky Mt. J. Math. 2019, 49, 681-697. [CrossRef]

26. Srivastava, H.M.; Ricci, P.E.; Natalini, P. A family of complex Appell polynomial sets. Rev. R. Acad. Cienc. Exactas Fis. Nat. Ser. A Mat. RACSAM 2019, 113, 2359-2371. [CrossRef]

27. Lehmer, D.H. Lacunary recurrence formulas for the numbers of Bernoulli and Euler. Ann. Math. 1935, 36, 637-649. [CrossRef]

28. Barman, R.; Komatsu, T. Lehmer's generalized Euler numbers in hypergeometric functions. J. Korean Math. Soc. 2019, 56, 485-505.

29. Komatsu, T. A parametric type of Cauchy polynomials with level 3. Miskolc Math. Notes, accepted for publication, MMN-3652.

30. Glaisher, J.W.L. Expressions for Laplace's coefficients, Bernoullian and Eulerian numbers etc. as determinants. Messenger (2) 1875, 6, 49-63.

31. Komatsu, T.; Ramirez, J.L. Some Determinants Involving Incomplete Fubini Numbers. An. Ştiinţ. Univ. “Ovidius" Constanţa Ser. Mat. 2018, 26, 147-153.

32. Muir, T. The Theory of Determinants in the Historical Order of Development; Dover Publications: New York, NY, USA, 1960 ; Volume 4. 
33. Trudi, N. Intorno ad alcune formole di sviluppo. Rendic. Dell' Accad. Napoli 1862, 135-143.

34. Brioschi, F. Sulle funzioni Bernoulliane ed Euleriane. Ann. Mat. Pura Appl. 1858, 1, 260-263. [CrossRef] 